Los Alamos National Laboratory, an alfirmative action/equal opportunity employer, is operated by the Unlversity of California for the U.S. Department of Energy under contract W-7405-ENG-36. By acceptance of this article, the publisher recognizes that the U.S. Government retains a nonexclusive, royalty-free license to publlsh or reproduce the publlshed form of this contribution, or to allow others to do so, for U.S. Government purposes. Los Alamos National Laboratory requests that the publisher Identify this article as work performed under the auspices of the U.S. Department of Energy. The Los Alamos National Laboratory strongly supports academic freedom and a researcher's right to publlsh; as an institution, however, the Laboratory does not endorse the viewpoint of a publlcation or guarantee its technical correctness. 


\section{DISCLAIMER}

This report was prepared as an account of work sponsored by an agency of the United States Government. Neither the United States Government nor any agency thereof, nor any of their employees, make any warranty, express or implied, or assumes any legal liability or responsibility for the accuracy, completeness, or usefulness of any information, apparatus, product, or process disclosed, or represents that its use would not infringe privately owned rights. Reference herein to any specific commercial product, process, or service by trade name, trademark, manufacturer, or otherwise does not necessarily constitute or imply its endorsement, recommendation, or favoring by the United States Government or any agency thereof. The views and opinions of authors expressed herein do not necessarily state or reflect those of the United States Government or any agency thereof. 


\section{DISCLAIMER}

Portions of this document may be illegible in electronic image products. Images are produced from the best available original document. 


\title{
III. ASTROPHYSICAL EQUATION OF STATE AND OPACITY
}

\author{
WERNER DÄPPEN \\ Department of Physics and Astronomy \\ University of Southern California \\ Los Angeles, CA 90089, U.S.A, \\ Theoretical Astrophysics Center, Aarhus University \\ 8000 Aarhus C, Denmark, and \\ Institute of Astronomy, Madingley Road, Cambridge, UK \\ AND \\ JOYCE A. GUZIK \\ Applied Theoretical and Computational Physics Division \\ XTA MS B220 \\ Los Alamos National Laboratory \\ Los Alamos, NM 87545, U.S.A.
}

\section{Introduction}

In stellar models, the equation of state and opacity are, together with nuclear reaction rates, the fundamental material properties. They have to be smooth, consistent, valid over a large range of temperatures and densities, and must incorporate the most important astrophysically relevant chemical elements. The equation of state appears as a necessary part of stellar modeling as well as of any opacity calculation. For the latter, it has to provide ionization equilibrium concentrations and level populations. However, the interest in the stellar equation of state is not merely motivated by astrophysics. It has turned out that one star - the Sun - is very special in two respects. First, the methods of helioseismology allow us to infer conditions in the solar interior very accurately (in particular, sound speed and density). Second, in the solar convection zone, helioseismology presents an opportunity to isolate the question of the equation of state from opacity and nuclear reaction rates, since the stratification is essentially adiabatic and thus determined by thermodynamics (Christensen-Dalsgaard \& Däppen 1992). Thus 
the Sun has become an astrophysical laboratory to study thermodynamic properties of a Coulomb system under conditions that cannot be achieved on Earth. Indirectly thus, the solar experiment also addresses a broader range of plasmas. In astrophysics, results for denser plasmas will have impact on models of Jupiter, Saturn, and probably brown and white dwarfs (Cauble et al. 1998), as well as of low-mass stars.

One of the major recent equation-of-state and opacity efforts is the international "Opacity Project" (OP; see the comprehensive books by Seaton 1995 and Berrington 1997). A necessary part of OP is the so-called MihalasHummer-Däppen (MHD) equation of state (cf. Section 2.3.3; Hummer \& Mihalas 1988; Mihalas et al. 1988; Däppen et al. 1987, 1988). However, the core of OP is a tremendous atomic data base, comprising state-of-the-art theoretical and experimental data. The MHD equation of state provides the ionization degrees of all astrophysically relevant chemical elements. The basic concept of the MHD equation of state was built on the heuristic idea of perturbed states of atomic, ionic, and molecular species. The usual ionization and dissociation reaction between these species are assumed; for this reason we speak of the so-called "chemical picture". At very high densities, when many-body effects become dominant, the concept of perturbed atoms loses its sense. For that reason, the MHD equation of state was originally restricted to the plasma of stellar envelopes, where density is sufficiently low that the concept of atoms makes sense. It turned out, however, that for the purpose of thermodynamic calculations at least, the aforementioned density domain was much too conservative. For instance, Christensen-Dalsgaard et al. (1988) applied the MHD equation of state to model the entire structure of the Sun and to predict solar oscillation frequencies. MHD remains a reliable tool down to the solar center, where density is about $150 \mathrm{~g} \mathrm{~cm}^{-3}$. The reason is that under those conditions, the plasma becomes virtually fully ionized. Therefore, in MHD, the limitations on the validity of the particular perturbation mechanism for bound species (Hummer \& Mihalas 1988) have little influence, simply because there are no bound species.

The other major recent equation-of-state and opacity approach, referred to as OPAL, is based on the so-called "physical picture" and provides a systematic method for including density effects. This effort is being pursued at Lawrence Livermore National Laboratory and is described by Iglesias \& Rogers $(1991,1993,1995,1996)$, Iglesias et al. $(1987,1992)$, Rogers \& Iglesias (1992, 1994), and Rogers et al. (1988). Its underlying equation of state is based on a sophisticated systematic method for including density effects. It starts out from the grand-canonical ensemble of a system of electrons and nuclei, which interact through the Coulomb potential (see Section 2.4 and Rogers 1977 1981, 1986, 1991, 1994, 1998; Rogers et al. 1996). Configurations corresponding to bound clusters of ions, atoms, and molecules 
appear at the appropriate stage of expansion in this ensemble (note that the grand-canonical involves a sum over all possible particle numbers). Any effects of the plasma environment on the internal states are obtained directly from the statistical mechanical analysis, rather than by assertion. In particular, the OPAL approach avoids the traditional ad hoc cutoff procedures of internal partition functions. The method employed in OPAL also provides a systematic procedure for including plasma effects on the photon absorption coefficients. So, for mixtures, OPAL does not follow an ideal-gas mixing procedure for combining the various photon absorption coefficients from the different elements. Instead, coupled equations for the full mixture are solved at each density and temperature point, removing thus a potential source of error.

Since the equation of state is a logical part of any opacity calculation, we begin with an introduction to equation of state issues, with emphasis on recent efforts to model fine details in order to match astrophysical challenges. Then, we present a review on the opacity of stellar matter. We show how new efforts have significantly improved the quality of stellar models. Applications to the particular case of the Sun are presented elsewhere in these proceedings [Christensen-Dalsgaard et al. 1999 (hereinafter Chapter II); see especially Sections II.4.3-II.4.5]. The relevance of the equation of state and opacity for stellar modeling in general is also discussed in these proceedings (Christensen-Dalsgaard \& Dziembowski 1999; hereinafter Chapter I). Since the equation of state is more basic, we will discuss it in considerable detail, while remaining more descriptive and summarizing in the opacity part.

The principal purpose of this chapter is to show what goes into equationof-state and opacity calculations. We believe that our information will help the user in choosing a specific equation of state and opacity. For this purpose we give at the end practical recommendations regarding the currently available equation of state and opacity data, including information about how to obtain them.

\section{The equation of state}

The simplest model is a mixture of nuclei and electrons, assumed fully ionized and obeying the classical perfect gas law. However, an ideal-gas equation of state can be more general. It may include deviations from the perfect gas law, namely ionization or dissociation reactions, radiation and degeneracy of electrons, as long as the underlying microphysics of these additional effects is still ideal, that is, does not contain interactions. The "particles", however, can be be classical or quantum, material or photonic. In such an ideal framework, bound systems (molecules, atoms, ions) are allowed to have internal degrees of freedom (excited states, spin). All such 
ideal effects can be calculated as exactly as desired.

One measure of nonideality in plasmas is the so-called coupling parameter $\Gamma$ [astrophysicists should note that $\Gamma$ has nothing to do with the adiabatic gradient $\Gamma_{1}$ introduced in Chapter I, Eq. (14)]. In a plasma of temperature $T$ and density such that particles of charge $e$ have an average distance $\langle r\rangle$ from each other, we can define $\Gamma$ as the ratio of average potential binding energy over mean kinetic energy $k_{B} T$ ( $k_{B}$ being the Boltzmann constant)

$$
\Gamma=\left(e^{2} /<r>\right) / k_{B} T
$$

In this expression we have for simplicity restricted ourselves to the case of hydrogen; generalizations to other elements are straightforward. Plasmas with $\Gamma \gg 1$ are strongly coupled, those with $\Gamma \ll 1$ weakly coupled. A famous example of a strongly coupled plasma is the electron gas in the interior of white dwarfs, where the coupling can become strong enough to force crystallization. Another example is given by the electrons in the conduction band of a metal at room temperature. Weakly coupled plasmas are, for instance, the interiors of stars with masses ranging from the slightly sub-solar ones to the largest.

\subsection{BASIC CONCEPTS AND DIMENSIONAL PARAMETERS}

There are two basic approaches to realize nonideal equations of state: the so-called chemical and physical pictures. In the chemical picture one assumes that the notion of atoms and ions still makes sense, and ionization is treated like a chemical reaction. Typically, as in the MHD equation of state, modifications of atomic states are expressed in a heuristic and intuitive way, by the probability that the state is occupied as a function of the parameters of the surrounding plasma.

In contrast, the physical picture provides a systematic method to include nonideal effects. It models an ensemble of a system of the basic constituents (electrons and nuclei), interacting through the Coulomb potential. Typically, as in the OPAL equation of state, the grand-canonical partition function is computed. Configurations corresponding to bound combinations of electrons and nuclei, such as ions, atoms, and molecules, arise naturally as terms in cluster expansions. Any effects of the plasma environment on the internal states are then obtained directly from the statistical-mechanical analysis, rather than by assertion as in the chemical picture.

To describe a degree of "nonideality" or "degeneracy" of the plasma, it is convenient to introduce a few dimensionless parameters to express the relative strength of the particular effects, such as the Coulomb interaction 
or quantum degeneracy. These parameters are defined as combinations of various "characteristic lengths" resulting from simple physical estimates.

The strength of the Coulomb interaction is given by the density-independent Landau length $l_{L}$

$$
l_{L}=\frac{e_{i} e_{j}}{k_{\mathrm{B}} T} .
$$

Although the Coulomb potential has infinite range, the Landau length represents an effective radius of the interaction between particles $i$ and $j$, i.e. for distances $\gg l_{L}$ the potential energy is negligible compared to the thermal energy $k_{\mathrm{B}} T$.

The Landau length clearly reflects a degree of nonideality of the plasma and the case of an "ideal" plasma corresponds to $l_{L}=0$.

Another purely classical characteristic length is the mean distance between two particles of the same species $\mathrm{k}(\mathrm{k}=\{e, i\})$

$$
d_{k}=\left(\frac{3}{4 \pi n_{k}}\right)^{1 / 3}
$$

Here, $n_{k}$ is the particle density $N_{k} / V$ of species $k$. Note that $d_{k}$ is the generalization of the quantity $\langle r\rangle$ in Eq. (1). It does not depend on temperature.

The most evident quantum mechanical characteristic length is the thermal de-Broglie wavelength

$$
\lambda_{k}=\frac{h}{\sqrt{2 \pi m_{k} k_{\mathrm{B}} T}} .
$$

The limits of applicability of various theoretical models of the equation of state are usually described by different dimensionless parameters. The degeneracy of species. $k$

$$
n_{k} \lambda_{k}{ }^{3}
$$

is very closely related to the well-known degeneracy parameter $\eta$ (sometimes also denoted $\psi$ ) by

$$
F_{1 / 2}(\eta)=\frac{\sqrt{\pi}}{4} \frac{\lambda_{e}{ }^{3} N_{e}}{V},
$$

where $F_{i}$ is the Fermi integral

$$
F_{i}(y)=\int_{0}^{\infty} x^{i}(1+\exp (y+x))^{-1} d x
$$


In the case of a stellar plasma with heavy nuclei and light electrons, the heavy particles become degenerate only at much higher densities than electrons.

\subsection{SCREENING AND THE DEBYE-HÜCKEL APPROXIMATION}

All characteristic lengths defined so far demonstrate either purely densityor purely temperature-dependent behaviour. No collective behaviour of particles has been considered. Here we discuss screening, which for normal stars is the most important deviation from ideality.

The seminal study of collective behavior in a plasma was the Debye \& Hückel (1923) theory of electrolytical solutions. Despite its phenomenological approach this theory proved to be immensely successful not just for electrolytes, but also for plasma calculations.

Basically, one mixes two ideas. First, assuming nondegenerate electrons, and considering one particular ion being fixed, the mean electron distribution around that ion is given by the Boltzmann factor

$$
n_{e}(r)=n_{e} \exp \left[\frac{+e \phi(r)}{k_{\mathrm{B}} T}\right]
$$

The mean ion density around the same ion is similarly given by

$$
n_{i}(r)=n_{i} \exp \left[\frac{-e \phi(r)}{k_{\mathrm{B}} T}\right]
$$

Second, one assumes applicability of Poisson's equation for mean charge distributions (instead of point charges)

$$
\Delta \phi(r)=-4 \pi e\left[n_{i}(r)-n_{e}(r)\right] .
$$

The resulting system of Eqs. (8) to (10) is complicated and nonlinear; however, if the system is only slightly nonideal, that is, if $e \phi(r) / k_{\mathrm{B}} T<1$, the linearized system has the well-known solution of the static-screened Coulomb potential (SSCP)

$$
\phi(r)=\frac{e}{r} e^{-\kappa r},
$$

where $\kappa$ is the reciprocal of the Debye-length $r_{D}$

$$
r_{D}=\sqrt{\frac{k_{\mathrm{B}} T}{4 \pi\left(n_{e} e^{2}+n_{i} e^{2}\right)}} .
$$

Having established the Debye radius, we can introduce a further dimensionless quantity, the diffraction parameter of species $k$

$$
\gamma_{k}=\frac{\lambda_{k}}{r_{D}}
$$


where $\lambda_{k}$ is again the thermal de-Broglie wavelength. The diffraction parameter refers to the relatively small quantum corrections to screening (cf. Brüggen \& Gough 1999, who have performed a nonlinear quantum mechanical screening calculation in the context of screened nuclear reaction rates). And yet, helioseismology has attained such a degree of precision that such small corrections to sound speed have become observationally accessible (see Chapter II, Section 4.3.2)

\subsection{CHEMICAL PICTURE. FREE-ENERGY-MINIMIZATION METHOD}

\subsubsection{Theory}

The development of specific methods in the chemical picture was strongly stimulated by needs for "engineering-type" equations of state, especially for stellar evolution, where data for a wide range of parameters are needed. The free energy minimization method, pioneered by G. Harris (Harris 1959, 1962; Harris et al. 1960) and especially its modern versions (e.g., Graboske et al. 1969; Däppen 1980) were very successful as a tool for practical equations of state.

The idea behind this method is simple and elegant. Given a mathematical model for the Helmholtz free energy $F\left(T, V,\left\{N_{i}\right\}\right)$, where $\left\{N_{i}\right\}$ is a set of particle numbers for all species $i$ present in a plasma, one minimizes $F$ subject to the stoichiometric relations governing possible reactions among the particle species in the plasma. The underlying principle is that nature adjusts the reaction equilibrium such that entropy is maximum (for given energy) or the free energy is minimum (for given temperature).

One starts from the canonical partition function. Consider a physical system (with Hamiltonian $H$ ) confined in a box of volume $V$ in contact with a heat reservoir at temperature $T$. Then the canonical partition function is a trace (denoted by $\mathrm{Tr}$ )

$$
\mathcal{Z}=\operatorname{Tr}\left(e^{-H / k_{\mathrm{B}} T}\right)
$$

From that, the free energy is obtained by the formula

$$
F(T, V,\{N\})=-k_{\mathrm{B}} T \ln (\mathcal{Z}) .
$$

Here, the volume $V$ is implicitly contained in the Hamiltonian operator. The free energy is calculated for all $\{N\}$ which are allowed by the stoichiometric relations and a given initial composition. Equilibrium concentrations are those $\{N\}$ that minimize $F(T, V,\{N\})$ for given $T, V$ under the stoichiometric constraints. This gives ionization and dissociation equilibria. Numerically, this will be a task of finding a minimum of a nonlinear function $F(T, V,\{N\})$ in the $\{N\}$-space under the stoichiometric constraints. 
The major assumption in any practical realization of the free-energy minimization method is that the total partition function $\mathcal{Z}=\mathcal{Z}_{\text {total }}$ factorizes, that is,

$$
\mathcal{Z}_{\text {total }}=\mathcal{Z}_{e} \mathcal{Z}_{\text {trans }} \mathcal{Z}_{\text {conf }} \mathcal{Z}_{\text {int }}
$$

where $Z_{e}$ here stands for the electronic contribution, $\mathcal{Z}_{\text {trans }}$ is the result of the integration over momentum space for the heavy particles, $\mathcal{Z}_{\text {conf }}$ comes from the integral over configuration space, and $\mathcal{Z}_{\text {int }}$ finally is

$$
\mathcal{Z}_{\text {int }}=\Pi \mathcal{Z}_{\text {int }}^{(i)}, \quad \mathcal{Z}_{\text {int }}^{(i)}=\sum_{j=1}^{\infty} g_{i j} \exp \left(-E_{i j} / k_{\mathrm{B}} T\right),
$$

where $E_{i j}$ and $g_{i j}$ denote energy and degeneracy of the state $j$ of species $i$, respectively.

When going from the partition function to the free energy, the consequence of the assumed factorizability of the partition function is additivity, or modularity, of the free energy. This modularity accounts for the great appeal of the chemical approach from both a modelistic and a computational point of view. Modularity basically tells us that all the interactions can be split into separate parts with clear physical meaning. And if, for example, one part needs to be modified to implement a higher order correction, it can be done without having to worry about consistency with the other parts. Terms can be introduced simply by adding or changing subroutines in a computer program, without a major overhaul of the previous work. That is why sometimes for the sake of modularity even relatively crude approximations are maintained, because a rigorous treatment, in the framework of the exact nonfactorizing partition function, would be entirely prohibitive.

\subsubsection{A simple application: The Saha equation}

By retaining only ideal terms, the free-energy-minimization method falls back to the time-honored Saha equation. To illustrate, consider the simplest case, hydrogen ionization $\left(\mathrm{H} \leftrightarrow \mathrm{H}^{+}+\mathrm{e}^{-}\right)$. A neutral mixture of $\mathrm{H}$-atoms, protons and electrons with particle numbers of $N_{\mathrm{H}}, N_{\mathrm{p}}$ and $N_{\theta}$, respectively, has only one degree of freedom, because of the two relations $\left(N_{\mathfrak{H}}^{0}\right.$ being the total number of protons, bound and free)

$$
\begin{gathered}
N_{\mathrm{H}}+N_{\mathrm{p}}=N_{\mathrm{H}}^{0}=\text { const }, \\
N_{\bullet}=N_{\mathrm{p}} .
\end{gathered}
$$

The degree of freedom is the ionization degree. Its equilibrium value is the one for which the free energy is minimum. The minimum condition is then 


$$
\frac{N_{H}}{N_{p} N_{e}}=\frac{\lambda_{e}^{3}}{V} \mathcal{Z}_{\text {int }}^{\mathrm{H}}=\sum_{j=0}^{\infty} g_{j} \exp \left(-\frac{E_{j}}{k_{\mathrm{B}} T}\right)
$$

(with $E_{j}$ and $g_{j}$ denoting the negative energy states of hydrogen and their orbital - statistical weight, respectively). This is the well-known Saha equation in astrophysics (known as the law of mass-action in physical chemistry). The infinity of the partition function in Eq. (20) is notorious; it has plagued statistical mechanics of plasmas in the last 70 years. The usual argument goes that excited states of hydrogen atoms become infinitely big with increasing quantum number $j$. Because of this, Eq. (20) would only be valid for a single atom in the whole infinite universe. Remedies of the infinity have been involving more-or-less sophisticated truncations of Eq. (20) at some level; a judicious choice allows to model real, interacting plasmas (see the specific expressions employed by the MHD equation of state $[(25),(27)]$.

We note in passing that even the most radical truncation to the ground state of hydrogen (whose orbital statistical weight is one)

$$
\frac{N_{H}}{N_{p} N_{e}}=\frac{\lambda_{e}^{3}}{V} \exp \left(-\frac{E_{0}}{k_{\mathrm{B}} T}\right)
$$

is not devoid of problems, because as one sees easily it leads to spurious recombinations at high densities and relatively low temperatures. Specifically, in the center of the Sun, Eq. (21) would predict about $30 \%$ neutral hydrogen, which is clearly nonsensical for the prevailing density of $150 \mathrm{~g} \mathrm{~cm}^{-3}$. The reason of this pathology is that the Saha equation does not contain information about the size of the hydrogen atom. Bringing in correction terms that do contain atomic radii amounts to modeling so-called "pressure ionization". One way of doing so is by modifying the internal partition function, a technique realized, e.g., in the occupation probabilities of the MHD equation of state [cf. (25), (27)].

\subsubsection{Nonideal effects in the free-energy-minimization method}

Re-deriving the Saha equation (20) with a new method is no gain though. However, the power of the free-energy-minimization method is precisely that it allows to include nonideal effects consistently. The reason is that any modification to the thermodynamics is only made at one single place (the free energy). Since the ionization degree and all thermodynamic quantities follow from the free energy by purely mathematical steps, all these quantities are consistent with each other. For instance, they automatically obey all Maxwell relations. The corresponding shifts in the ionization equilibria are correct. 
Achieving such consistency would be difficult in an approach where one considers the Saha equation and the corresponding thermodynamic quantities separately, all to be modified individually. There would be no general systematic procedure. The free-energy-minimization method, however, achieves it nicely and simply. It is therefore the natural nonideal extension of equations of state that are based on the Saha equation.

As an example, to include screening at the level of the Debye-Hückel approximation, it suffices to add to the total free energy the "module" for the Debye-Hückel free energy (which can be found in many books on statistical mechanics, e.g. , in Reichl 1980)

$$
F_{\text {conf }} \equiv F^{D H}=-\frac{k_{B} T V}{12 \pi r_{D}^{3}}
$$

If the analysis of the free energy minimization method for a hydrogen plasma is repeated with this new $F_{\text {conf }}$ added, the final result can still be written in the form of equation (20), provided that $Z_{\text {int }}$ is replaced by

$$
Z_{\text {int }}=\sum_{j} g_{j} \exp \left[-\beta\left(E_{j}+e^{2} / r_{D}\right)\right] .
$$

The effect of the Coulomb correlations can be interpreted as a lowering of the continuum by the amount $e^{2} / r_{D}$ and, consequently, the ionization balance is shifted toward increased ionization (see also Baturin et al. 1996).

One way of introducing a possibility to treat pressure ionization is to assign "weights" or "occupation probabilities" to all the bound states of all the species. The internal partition function then becomes

$$
Z_{j k}^{*}=\sum_{i} w_{i j k} g_{i j k} \exp \left(-\beta E_{i j k}\right)
$$

Here $w_{i j k}$ is the probability that a state $i$ of ion $j$ of species $k$ still exists despite the plasma environment, and $g_{i j k} \exp \left(-\beta E_{i j k}\right)$ remains the probability that this state is actually occupied.

The occupation probability formalism has several advantages:

- The $w_{i j k}$ decrease continuously and monotonically as the strength of the relevant interaction increases.

- States fade out now continuously with decreasing $w_{i j k}$ and thus assure continuity not only of the internal partition function but also of all material properties (pressure, internal energy, etc.) In contrast, truncations of the sum in Eq. (20) that depend on the continuous parameters $T$ and $V$ have the unpleasant consequence that the partition function is subject to finite jumps for infinitesimal variations of the parameters. In other words, it is discontinuous. Thermodynamic deriva- 
tives would be thus cluttered with Dirac $\delta$-functions. Equation (24) avoids this problem.

- The $w_{i j k}$ can be made analytically differentiable, which permits use of a second-order convergent numerical scheme in the free-energy minimization.

For neutral perturbing species, Hummer \& Mihalas (1988) started out from a widely studied hard-sphere model (Fowler 1936) with each state in principle having its own diameter. However, the simple binary interaction model is computationally prohibitive because it accounts for perturbations from all ions of all chemical species in all possible excited states. That implies thousands of the individual occupation numbers $N_{i j k}$ as independent variables in the free energy minimization. In addition, in this case the function $f$ is nonlinear.

As an obvious first approximation, MHD considered the low-excitation limit (Hummer \& Mihalas 1988) in which it is assumed that essentially all perturbers encountered by an atom in an exited state reside in the ground state.

$$
\left(w_{i j k}\right)_{n e u t r a l}=\exp \left[-(4 \pi / 3 V) \sum_{j^{\prime}, k^{\prime}} N_{j^{\prime} k^{\prime}}\left(r_{i j k}+r_{1 j^{\prime} k^{\prime}}\right)^{3}\right] .
$$

One sees that in this approximation only the total occupation numbers (summed over the states $i$ ) enter, that is, $N_{j k}=\sum_{i} N_{i j k}$ for all species $j$ of chemical element $k$.

In the case of charged perturbing particles, MHD defines the $w_{i j k}$ directly, arguing that the presence of a plasma microfield destroys high-lying states by means of a series of Stark level mixing with higher lying states leading to the continuum.

The basic idea is that for each bound state $i$ of every unperturbed ion $j$ of element $k$, there is a critical value of the electric field $F_{i j k}$ such that the state in question cannot exist if the field exceeds the critical value. Then probability that a given state does exist is simply the probability that the field strength is less than $F_{i j k}$, i.e.

$$
\left(w_{i j k}\right)_{\text {charged }}=\int_{0}^{F_{i j k}} P(F) d F,
$$

where $P(F)$ is the microfield distribution function (i.e., the distribution of electrical field strength in the plasma). The choice of an appropriate plasma microfield $P(F)$ is not straightforward. Hummer \& Mihalas (1988) have made the following choice 


$$
\left(w_{i j k}\right)_{\text {charged }}=\exp \left\{-\left(\frac{4 \pi}{3 V}\right) 16\left[\frac{\left(Z_{j k}+1\right)^{1 / 2} e^{2}}{K_{i j k}^{1 / 2} \chi_{i j k}}\right]^{3} \sum_{j^{\prime}, k^{\prime}} N_{j^{\prime} k^{\prime}} Z_{j^{\prime} k^{\prime}}^{3 / 2}\right\},
$$

where $Z_{j k}$ denotes the charge of ion $j$ of chemical species $k$ (thus, zero for neutral particles) and the sum runs over all levels $i$ of ions $j$ of species $k$, and $K_{i j k}$ is the quantum correction factor of those levels (see eq. (4.70) of Hummer \& Mihalas 1988). We will show below (Section 3.2) that the approximations entering (27) (a simplified form of the Holtsmark $1919 \mathrm{mi}$ crofield distribution) are a source of concern for OP opacities. For this reason, very recently an upgraded version of the MHD equation of state with more realistic distribution functions has been developed (Nayfonov et al. 1999).

\subsection{PHYSICAL PICTURE. ACTIVITY EXPANSION}

It is clear from the preceding sections that the advantages of the freeenergy-minimization method and the chemical picture lie in the possibility to model complicated plasmas, and to obtain numerically smooth and consistent thermodynamical quantities. Nevertheless, the heuristic method of the separation of the atomic-physics problem from that of statistical mechanics is not satisfactory, and attempts have been made to avoid the concept of a perturbed atom in a plasma altogether. This has suggested an alternative description, the physical picture. In such an approach one expects that no assumptions about energy-level shifts or the convergence of internal partition functions have to be made. On the contrary, properties of energy levels and the partition functions should come out from the formalism.

There is an impressive body of literature on the physical picture. Important sources of information with many references are the books by Ebeling et al. (1976), Kraeft et al. (1986), and Ebeling et al. (1991). However, the majority of work on the physical picture was not dedicated to the problem of obtaining a high-precision equation of state for stellar interiors. Such an attempt was made for the first time by the OPAL group at Livermore as part of their opacity project (see Section 1 and references therein).

To explain the advantages of this approach for partially ionized plasmas, it is instructive to discuss the activity expansion for gaseous hydrogen. The interactions in this case are all short ranged and pressure is determined from a self-consistent solution of the equations (Rogers 1981)

$$
\frac{p}{k_{B} T}=z+z^{2} b_{2}+z^{3} b_{3}+\ldots
$$




$$
\rho=\frac{z}{k_{B} T}\left(\frac{\partial p}{\partial z}\right)
$$

where $z=\lambda_{e}^{-3} \exp \left(\mu / k_{B} T\right)$ is the activity [with $\lambda_{e}$ being the thermal deBroglie wavelength of electrons, see Eq. (4)], and $\mu$ is the chemical potential. The $b_{n}$ are cluster coefficients such that $b_{2}$ includes all two particle states, $b_{3}$ includes all three particle states, etc.

In contrast to the chemical picture, which is plagued by divergent partition functions, the physical picture has the power to avoid them altogether. An important example of such a fictitious divergence is that associated with the atomic partition function. This divergence is fictitious in the sense that the bound-state part of $b_{2}$ is divergent but the scattering state part, which is omitted in the Saha approach, has a compensating divergence. Consequently the total $b_{2}$ does not contain a divergence of this type (Ebeling et al. 1976; Rogers 1977). A major advantage of the physical picture is that it incorporates this compensation at the outset. A further advantage is that no assumptions about energy-level shifts have to be made; it follows from the formalism that there are none.

As a result, the Boltzmann sum appearing in the atomic free energy for atoms and compound ions is replaced with the so-called Planck-Larkin partition function (PLPF), given by e.g. Ebeling et al. (1976), Kraeft et al. (1986), or Rogers (1986)

$$
\text { PLPF }=\sum_{n l}(2 l+1)\left[\exp \left(-\frac{E_{n l}}{k T}\right)-1+\frac{E_{n l}}{k_{B} T}\right] .
$$

The PLPF is convergent without additional cut-off criteria as are required in the chemical picture. We stress, however, that despite its name the PLPF is not a partition function, but merely an auxiliary term in a virial coefficient (see, for example, Däppen et al. 1987).

An alternative approach based on the physical picture is the pathintegral based Feynman-Kac (FK) formalism of Alastuey \& Perez (1992, 1996), Alastuey et al. $(1994,1995)$. It allows to compute exact coefficients of density (virial) expansions. The application of this formalism to solar physics is in progress (Perez \& Däppen 1999).

\section{Opacity}

Opacity has long been an issue in understanding stars. As long ago as 1926, Eddington identified opacity as one of two clouds obscuring stellar model calculations (the other being the source of stellar energy). At that time it was thought that bound-bound absorption was not a significant source of opacity. It was another 40 years before Cox \& Stewart (1962; $1965 ; 1970 a, b)$ included bound-bound transitions and obtained increases 
in the Rosseland mean opacity exceeding a factor of three in some cases. The Cox-Stewart opacities greatly improved the quality of stellar models and remained the standard for more than a quarter century. This work continued to be modified and improved by Cox and others at Los Alamos ((Cox \& Tabor 1976); (Huebner et al. 1977)). A detailed description of this first generation Los Alamos opacity (LAOL) is given by Huebner (1986).

Even though the LAOL opacities helped elucidate many features of stars, a number of observations continued to resist explanation. For example, period ratios in classical Cepheid models were too low, the mechanism for pulsation in $\beta$-Cephei stars could not be identified, the calculated $\mathrm{Li}$ abundance in dwarf stars of the Hyades cluster was much less than observed, and simulations underestimated wind-driven mass loss in classical novae. A number of studies found that these problems are sensitive to changes in opacity (Fricke et al. 1971; Petersen 1974; Stellingwerf 1978). However, the opacity increases needed seemed unrealistically large, as much as $300 \%$ in the case of the classical Cepheids and the $\beta$-Cephei stars. Simon (1982) determined that increasing the opacity for temperatures above $1 \times 10^{5} \mathrm{~K}$ would be sufficient to resolve the Cepheid and $\beta$-Cephei problems. He speculated that problems with heavy element opacities could be responsible and issued a plea for their reinvestigation. A group at Los Alamos (Magee et al. 1984) was the first to respond. They concluded that such large increases in opacity were inconsistent with atomic physics. Nevertheless, two other groups started completely new efforts to calculate opacity.

As mentioned in the introduction, one of these is the Opacity Project (OP), led by M. Seaton at University College, London; the other is OPAL, an effort pursued at Lawrence Livermore National Laboratory. These efforts have obtained large increases in the opacity (Iglesias et al. 1987; 1992; Iglesias \& Rogers 1991; 1996; Rogers \& Iglesias 1992; Seaton et al. 1994) which helped resolve a number of long-standing puzzles (Rogers \& Iglesias 1994). The differences between OP and OPAL opacities are generally small compared with the differences between either OPAL or OP and the older LAO1. An important exception is with solar interior opacities where OPAL obtained modest increases over LAOL, while OP is $40 \%$ lower. The decrease seems incompatible with helioseismology (Bahcall \& Glasner 1994; Tripathy et al. 1997) and has been attributed to approximations in the OP calculations (Iglesias \& Rogers 1995). As we will show below (Section 4.3.2), a second generation of Los Alamos opacities (LEDCOP) have recently been released (Magee et al. 1998). And, as we will will further address in Section 4.4 , there have been several new efforts to calculate surface opacities in addition to the new stellar interior opacity calculations. 


\subsection{GENERAL FEATURES OF OPACITY CALCULATIONS}

In the solar interior the photon mean-free-path is sufficiently small so that radiation transport occurs via diffusion. When the diffusion approximation is valid, the Rosseland mean $\kappa_{R}$ over the monochromatic opacity $\kappa_{\nu}$, defined by

$$
\frac{1}{\kappa_{R}}=\frac{\pi}{4 \sigma T^{3}} \int_{0}^{\infty} \frac{1}{\kappa_{\nu}}\left(\frac{\partial B}{\partial T}\right)_{\nu} d \nu
$$

guarantees the correct integrated flux transport. Here, $\sigma$ denotes the StefanBoltzmann constant and $B(T, \nu)$ the Planck function.

\subsubsection{Atomic Models and Categories of Approximations}

The atomic data relevant for opacity calculations are in general influenced by the plasma interactions which modify the interatomic potential and wavefunctions, and therefore energy levels, level widths, transition moments, spectral line shapes, etc. In the most general formulation, the system is the atom (or ion) and the plasma. This is a very complicated many-body problem, but for low densities, one can resort to a perturbative approach, in which an isolated atom (or ion) is modified with plasma "corrections" to energy levels, widths, etc. It is important to note that these plasma corrections effectively limit the number of bound states which would otherwise exist.

Energy levels of atomic configurations are characterized according to the level of approximations. In the central field approximation, the pair of indices $n l$ completely specifies the electron configuration in the absence of electrostatic effects between electrons. Inclusion of the electrostatic effects and the spin-orbit interaction splits the energy levels into a number of sublevels. When the electrostatic interaction dominates over spin-orbit, we have the familiar case of $L S$ coupling. In the opposite case, we have the case of $j j$ coupling. Although the spin-orbit interaction increases in importance with increasing atomic charge $Z$, the occurrence of pure $j j$ coupling is relatively rare, with the intermediate case (intermediate coupling) being more common. Highly excited states in noble gas atoms often experience intermediate coupling. However, in many analyses $L S$ coupling is assumed because of its historical importance in the analysis of light-element spectra.

The atomic models may be divided into two broad categories: averageatom models and detailed configuration-accounting models. In the averageatom model, a mean-field approximation is used to calculate the (fictitious) fractional occupancy of the respective one-electron energy levels. This provides an immediate gross indication of plasma ionization and excitation. An application of first-order perturbation theory allows a deconvolution to the detailed ionic species and excited configurations. Historically, this method 
has been used at high temperatures $\left(k_{B} T>100 \mathrm{eV}\right)$. The method of detailed configuration accounting uses either experimental data or ab initio data (or a mixture of both) for the individual energy levels for all ions. A more extended discussion of these approaches can be found in Cox (1965) and Huebner (1986).

Contributions to the opacity arise from several distinct physical processes: bound-bound transitions, bound-free (that is, photoionization), freefree (that is, bremsstrahlung), and scattering. For sufficiently large densities, electron conduction can also contribute. The following subsections only serve to review the principal methods and results. More details can be found in the references cited.

\subsubsection{Bound-Bound Transitions}

The spectrum arising from the totality of all spectral-line absorptions has a profound effect on the radiative opacity. Since the Rosseland mean opacity is a harmonic mean, it is especially sensitive to the windows arising from the distribution of transition arrays (the totality of transitions between two configurations), and to the windows arising within the transition arrays. While the distribution of transition arrays and line patterns within the arrays is determined by the atomic model, the line-profile functions are also crucial in determining the overall intensity distribution.

In addition to the natural broadening that arises from the finite lifetime of a radiating atom with a Lorentz profile

$$
\Phi_{\text {nat }}\left(\nu, \nu_{0}\right)^{2}=\frac{\left(\frac{\Gamma}{2 \pi}\right)^{2}}{\left(\frac{\Gamma}{2 \pi}\right)^{2}+\left(\nu-\nu_{0}\right)^{2}},
$$

and to Doppler broadening that arises from the thermal ensemble of radiating atoms which has a Gaussian distribution

$$
\Phi_{\text {therm }}(v)=\sqrt{\frac{\pi m}{2 k_{B} T}} \exp \left(-\frac{m v^{2}}{2 k_{B} T}\right),
$$

there is also collision broadening, whose nature depends explicitly on the nature of target and perturber.

Further line broadening occurs through electron impacts on compound objects (atoms or ions), which result in shifted Lorentz profiles, approximately evaluated classically (Lindholm 1946; Foley 1946). Quantum effects (linear and quadratic Stark effect; excited states) are important (Griem et al. 1962). Assuming that natural and electron-impact broadening are uncorrelated, their Lorentzians can be convoluted to yield a Lorentzian whose damping width is the sum of the individual damping widths. A subsequent 
convolution with the thermal Gaussian yields the so-called "Voigt profile", which can be rather time consuming to evaluate in the case of absorption spectra involving millions of lines (Hui et al. 1978).

\subsubsection{Bound-Free Absorptions}

In a partially ionized gas, photoionization is an important component of the absorption. The earliest result for photoionization is the semi-classical result by Kramers

$$
\sigma_{\mathrm{bf}}=\frac{2^{6} \pi^{4}\left(Z^{*}\right)^{4} m e^{10}}{3 \sqrt{3} \mathrm{ch}^{6} n^{5} \nu^{3}} g_{\mathrm{bf}}\left[\mathrm{cm}^{2}\right]
$$

( $Z^{*}$ being an effective charge), with its characteristic frequency dependence, and to which quantum mechanical corrections, the so-called "Gaunt factors" are incorporated in the form of the factor $g_{\mathrm{bf}}$ (detailed references can be found in Däppen et al. 1991, p. 132).

Nowadays, atomic models, based on e.g. Hartree-Fock or close-coupling methods, are nowadays being used to calculate energy levels and oscillator strengths. They allow us to calculate the photoionization spectra directly, assuring continuity in the absorption strength across the Rydberg series into the continuum.

\subsubsection{Free-Free Absorptions}

A free electron in the field of an atom or ion can absorb a photon (inverse bremsstrahlung) or emit one bremsstrahlung. A frequently used form is again that of a product of the Kramers cross section $\sigma_{\mathrm{K}}$ with a Gaunt factor $\left(g_{\mathrm{ff}}\right)$

$$
\sigma_{\mathrm{ff}} \equiv \sigma_{\mathrm{K}} g_{\mathrm{ff}} \equiv \frac{2^{4} \pi^{2}\left(Z^{*}\right)^{2} e^{6}}{3 \sqrt{3} h \mathrm{c}(2 \pi m)^{3 / 2}} \frac{N_{e}}{\sqrt{k_{B} T}} \frac{g_{\mathrm{ff}}}{\nu^{3}}\left[\mathrm{~cm}^{2}\right]
$$

As for the case of bound-free absorptions, modern atomic-structure calculations permit a direct calculation of $\sigma_{\mathrm{ff}}$. Excitation effects during the scattering processes can give relevant corrections, but they have not yet received much attention. In partially ionized plasmas, the free-free part is seldom dominant in the Rosseland mean, although it dominates the lowfrequency monochromatic absorption. At very high temperatures (above about $1 \mathrm{keV}$ ), where the gas is dense and nearly fully ionized, the free-free process with hydrogen ions is important, but the aforementioned treatment of equation (34) should be accurate.

\subsubsection{Scattering}

The Thompson formula 


$$
\sigma_{\mathrm{T}}=\frac{8 \pi e^{4}}{3 m^{2} c^{4}}
$$

describes the behavior of photons scattering off both bound and free electrons. For conditions of the solar center, high-energy effects (Compton regime) can be incorporated using the Klein-Nishina formula. Since this formulation is based on the rest frame of the fast electrons, a correction must take into account the observer's (laboratory) frame (for references see Däppen et al. 1991, p. 133). Finally, electron correlation effects [Diesendorf \& Ninham 1969; Watson 1969; Huebner 1986 (especially Eq. 85)] are important if the product of the photon wavenumber and the Debye radius is approximately equal to or less than unity. For a discussion of further nuances in the scattering process, see Boercker (1987).

\subsection{SPECIFIC FEATURES OF THE OPAL AND OP CALCULATIONS}

As we have seen in the previous subsection, the calculation of opacity involves four distinct disciplines: equation of state, atomic physics, spectral line broadening, and plasma collective effects. The older LAOL opacities were calculated with an ad hoc model of the equation of state and mostly hydrogenic approximations to the atomic physics. The new OP and OPAL opacity efforts are based on improved theoretical methods in all four of the disciplines mentioned above. In the following the improved physics and its impact on opacity are briefly described. More detailed accounts can be found in Rogers \& Iglesias (1992), Iglesias \& Rogers (1996), Rogers (1998), and in the books by the OP team (Seaton 1995; Berrington 1997).

Although differences in equation of state models have in general not significantly affected astrophysical opacities, differences in bound state occupation numbers are a primary reason for OPAL opacity enhancements near the base of the solar convection zone (Iglesias \& Rogers 1991). It is also one of the reasons OP obtains a smaller opacity than LAOL and OPAL in this region (Iglesias \& Rogers 1995). For this reason, the MHD equation of state has been upgraded to include more realistic distribution functions (Nayfonov et al. 1999).

By far the most significant effect on opacity has come from improved calculations of bound-bound absorption that include much more detailed atomic data. The OPAL and OP groups chose different approaches for this part of the calculation. The goal of OPAL was solely to calculate opacity, whereas OP had the additional aim to produce a general purpose atomic database. A continuation of that effort known as the Iron Project is still in progress (Bautista \& Pradhan 1997). For the required atomic data the OPAL group developed a parametric potential method that is fast enough 
to allow on-line calculations, while achieving accuracy comparable to single configuration Dirac-Fock self-consistent field calculations (Rogers et al. 1988). This on-line capability provides flexibility to study easily the effects of atomic physics approximations; e.g. angular momentum coupling or data averaging methods. By contrast, the OP group uses first principle (nonrelativistic) methods to construct detailed atomic databases (Seaton 1987; Seaton et al. 1994). The large increase in the iron opacity obtained with the LS coupling scheme compared to calculations that neglect term splitting suggested that fine structure is also important ( Rogers \& Iglesias 1992). OPAL opacities calculated since 1992 include spin-orbit effects in full intermediate coupling (Iglesias et al. 1992), while OP (Seaton et al. 1994) uses an approximate method that does not include spin changing transitions (see Fig. 1 of Rogers \& Iglesias 1994). On the other hand, the OPAL calculation assumes single configurations, while OP includes configuration-interaction effects in both the bound-bound and bound-free calculations. Configuration-interaction is most important for atoms and near neutral ions.

The OPAL calculations include degeneracy and plasma collective effects in the free-free absorption using a screened form of the parametric potentials, whereas these effects are neglected in OP. Both OPAL and OP include collective effects in Thomson scattering (Boercker 1987). The OPAL spectral line broadening for one, two, and three electron ions is computed with a suite of codes provided by Lee (1988) that include linear Stark theory. For all other transitions the OPAL calculations use Voigt profiles where the Gaussian width is due to Doppler broadening and the Lorentz width is due to natural plus electron impact collision broadening (Dimitrievic \& Konjevic 1980). The OP approach is similar (Seaton 1987) except that for spectral lines not subject to linear Stark effect OP uses widths from quantum-mechanical close coupling calculations (Seaton 1988), which are similar to those used by OPAL.

The improved line broadening has in general had a small effect on opacity. One important exception is Stark broadening of hydrogen. LAOL used the theory of Griem (1960) which gives lines that are much too broad compared to experiment (Wiese et al. 1972). The OP and OPAL hydrogenic lines agree well with the data and result in an opacity reduction for Population II compositions around $\log T=4.8$. Cox (1991) showed that this reduction in opacity in conjunction with a modest increase in opacity for $\log T \approx 5.3$ removes several long-standing puzzles in models of RR-Lyrae stars.

An important source of discrepancy between OP and OPAL has been traced to an approximation in OP affecting the occupation numbers. Hummer \& Mihalas (1988) assumed that the Holtsmark electric microfield, valid 
for randomly distributed ions, determines the probability a state is localized. Furthermore, in order to reduce computational expense they adopted an approximate form of the Holtsmark function. In a real plasma however, the Coulomb interaction modifies the ion distribution and causes the microfield distribution to peak at lower values of the field strength relative to Holtsmark. Consequently, the probability that a state is dissolved by the electric microfield fluctuations is reduced. Iglesias \& Rogers (1995) show: 1) the OP approximation to Holtsmark is poor; 2) using the more realistic APEX microfield (Iglesias et al. 1985) significantly increases the OP occupation numbers (as defined in Rogers 1986) for high lying states, bringing them closer to OPAL. The recently upgraded MHD equation of state includes post-Holtsmark distribution functions (Nayfonov et al. 1999), and it should lead to higher OP values. Since the Hummer \& Mihalas procedure and OPAL are based on different physical assumptions, the two calculations can of course not be expected to agree exactly.

\section{Practical Recommendations}

\subsection{INTRODUCTION}

When constructing a stellar evolution or pulsation code, opacity and equation of state formulations are necessary. These may be in the form of analytical or in-line routines that calculate the desired opacities or thermodynamic quantities, given as input the temperature, density, and chemical composition. Pre-calculated opacity or equation of state (hereafter EOS) tables may also be used, with appropriate look-up and interpolation routines. Sometimes several tables or analytical fits covering different regions of $T$, $\rho$, and composition space must be smoothly joined. The hydrostatic structure calculation may require fewer thermodynamic quantities or derivatives than a pulsation calculation; for example, in pulsation calculations, smooth derivatives of opacity with respect to temperature and density, as well as additional derivatives of thermodynamic quantities are required.

There are advantages and disadvantages of using tables versus in-line analytical formulations. Tables are often limited in coverage of $T, \rho$ and composition space, and so may not be useful for stellar models over a wide range of masses, compositions, and evolutionary states. Tables do not always include all of the thermodynamic quantities needed for a calculation, and these quantities cannot always be constructed from the information provided. Interpolation within and between tables, or extrapolation off the edges of tables can be difficult and risky, and introduces significant uncertainty into the calculations (see, e.g., Christensen-Dalsgaard \& Däppen 1992 and Houdek \& Rogl 1993). The grids of table entries are not always fine enough for good interpolation, or for construction of derivatives using 
differences between table quantities. On the other hand, tables often include superior physics compared to simple analytical schemes, and can be less computationally expensive than a sophisticated analytical routine.

Analytical procedures can be more flexible, for example in taking into account variations in element mixtures and composition, or in providing smooth derivatives. They also may be modified easily to generate additional thermodynamic quantities. Analytical procedures can also be useful to explore the importance of various physical processes, since these processes can easily be turned on and off, exaggerated with multipliers, etc.

Below we summarize some commonly used and available analytical and table opacities and equations of state. We also list a few papers in which these equations of state and opacities have been tested and compared against the stringent constraints of helioseismology, or in application to other types of stars, e.g. low-mass stars and white dwarfs.

\subsection{EQUATION OF STATE}

Well into convection zones, where convection is very efficient and carrying nearly all of the stellar luminosity, as long as the opacity is realistic, opacity plays no role in stellar structure. An example is in the solar envelope convection zone, where helium is ionizing. In these regions, the EOS alone determines the sound speed and the hydrostatic structure. For this reason, helioseismology can be a sensitive test of equation of state treatments, and also can be used to determine abundances of elements such as He ionizing in the solar envelope.

Note that some table-based and analytical equations of state return the pressure and energy only for the gas. The user should check this point, and self-consistently include the radiation pressure $\left(1 / 3 a T^{4}\right)$ and energy to obtain total pressure and energy. Care should also be taken to include the radiation pressure and energy in the derivative quantities.

\subsubsection{Analytical Equations of State}

a) Ionizing Perfect Gas Mixture

See Cox \& Giuli (1968), section 9.18. Cox \& Giuli describe a basic EOS formulation for stellar applications, giving thermodynamic quantities for a mixture of perfect gases, ignoring relativistic and degenerate effects, but including radiation pressure, using the simple Saha ionization equation. Stellingwerf (1975a) describes his implementation whereby, given $\mathrm{T}$ and $\rho$, he solves iteratively for the mean molecular weight per free electron, from which all other necessary thermodynamic quantities follow. Stellingwerf assumes that all elements heavier than helium ionize when helium does. 


\section{b) $\mathrm{EFF}$}

The Eggleton, Faulkner \& Flannery (1973) EOS is relatively simple and easy to program. EFF is thermodynamically consistent, and includes a pressure ionization treatment that is ad hoc but qualitatively correct. EFF should not be used for low-mass stars, as it introduces pseudo phase transitions and multivalued state functions. However, this EOS does include relativistic effects, which were omitted by the more modern MHD and OPAL tables. EFF does not include excited states, $\mathrm{H}$ molecule formation, or Coulomb corrections, and treats heavy elements as fully-ionized (see also Table 1 below). Iben (1975) adopted this equation of state as an option for stellar interiors where the assumption of complete ionization is reasonably valid.

c) Vardya EOS

Vardya (1964) developed a simple analytical procedure for including the effects of partial ionization. He includes ionization of $\mathrm{H}$ and $\mathrm{He}$, plus one electron from a representative heavier ionic species with electron potential of $7.5 \mathrm{eV}$. Despite its crudeness, this EOS does a surprisingly good job, as evidenced by solar model comparisons with the modern MHD EOS (Guzik \& Cox 1991), because heavy elements of a solar mixture contribute less than $1 \%$ of the free electrons that generate the pressure. Iben (1975) adopted this equation of state for stellar envelope calculations.

\section{d) CEFF}

CEFF (Christensen-Dalsgaard \& Däppen 1992) is the EFF EOS with the addition of a thermodynamically consistent treatment of Coulomb corrections. Christensen-Dalsgaard \& Däppen introduced the Coulomb term in the Debye-Hückel approximation taken from the MHD EOS. This analytical EOS has been popular for helioseismic applications. CEFF is available from J. Christensen-Dalsgaard (e-mail: jcdQobs.aau.dk).

\section{e) SIREFF}

The Swenson, Irwin, Rogers, Eggleton, Faulkner \& Flannery (Guzik \& Swenson 1997) in-line equation of state is based on the EFF EOS, but includes many additional refinements (See Table 1 below). Like MHD, this EOS is derived in the chemical picture (see 2.3). It has nearly all of the physics (even some additional) that are contained in the most modern tabular equations of state (MHD and OPAL). However, it also has the advantages of an analytical EOS, such as the flexibility to allow variable element abundances and mixtures that may develop within a stellar model due to element diffusion, radiative levitation or nuclear processing. Another advantage of SIREFF is that it accounts for $\mathrm{H}_{2}$ molecules, and so gives better 
results in principle than OPAL for temperatures $\leq 6000 \mathrm{~K}$. Like EFF, SIREFF includes relativistic effects, which turn out to be non-negligible in the solar core (Elliot \& Kosovichev 1998).

SIREFF is more computationally intensive than EFF, but not so intensive as directly calculating new MHD or OPAL table points for many new mixtures. This EOS includes a new generalized pressure ionization treatment that is still ad hoc, but is a function of ion density in addition to electron density. The adjustable parameters in the pressure ionization treatment have been calibrated by detailed comparison to the OPAL EOS for the run of interior $\mathrm{T}, \rho$ of a solar model and a $0.3 \mathrm{M}_{\odot}$ model. SIREFF is written in FORTRAN, and is available from J. A. Guzik or F. J. Swenson (e-mail: joyolanl.gov). Note also that (Irwin et al. 1999) are developing an in-line EOS including excited states, more molecules, and an improved Coulomb treatment that is available for testing as of this writing.

\subsubsection{Equation of State Tables}

\section{a) MHD EOS}

The MHD EOS (Hummer \& Mihalas 1988; Mihalas et al. 1988; Däppen et al. 1987, 1988). was developed as part of the OP project, and is based on the chemical picture. See Table 1 below for a summary of the physical refinements included.

One advantage of the MHD tables over the OPAL set is that they include hydrogen molecules $\left(\mathrm{H}_{2}, \mathrm{H}_{2}^{+}\right.$, and $\left.\mathrm{H}^{-}\right)$, and so they are available and, in principle, valid to temperatures lower than the OPAL tables $(<6000 K)$. The MHD tables have been validated for stellar masses as low as $0.4 \mathrm{M}_{\odot}$ (Charbonnel et al. 1999 ), whereas the OPAL tables claim validity for $M>0.8 \mathrm{M}_{\odot}$.

The first MHD tables computed for solar applications included the elements $\mathrm{H}, \mathrm{He}, \mathrm{C}, \mathrm{N}, \mathrm{O}$, and Fe. Eight tables were provided: Three "ZAMS tables" with $\mathrm{X}=0.69,0.72,0.75$, and five "interior tables" with $\mathrm{X}=0.31$, $0.42,0.53,0.64$, and 0.75 . In those early versions of the MHD tables, the element composition was chosen to be $Z=0.02$, and the heavy-element distribution was based on the Ross \& Aller (1976) solar mixture (with iron representing all elements not included in the mixture). More recent MHD tables are based on the Grevesse \& Noels (1991) mixture. They contain the elements $\mathrm{H}, \mathrm{He}, \mathrm{C}, \mathrm{N}, \mathrm{O}$, and $\mathrm{Ne}$, with $\mathrm{Ne}$ representing all other elements. This is precisely the mixture used for current OPAL equation-of-state tables. These newer tables are therefore specifically adequate for equation-ofstate comparisons. Recently, $\mathrm{Z}=0.001$ tables have been computed for use in the Geneva-group low-mass stellar models (Charbonnel et al. 1999).

A simple MHD table that contains a sample of ionization fractions and thermodynamic quantities can be found at the OP web site 
TABLE 1. Fine effects included in EFF, MHD, OPAL, and SIREFF EOS

\begin{tabular}{lllll}
\hline Effect & EFF & MHD & OPAL & SIREFF \\
\hline Coulomb Correction & No & Yes $^{a}$ & Yes & Yes $^{a}$ \\
Pressure Ionization & Yes $^{b}$ & Yes & Yes & Yes $^{c}$ \\
Partial Ionization & Yes & Yes & Yes & Yes \\
Molecules & No & $\mathrm{H}_{2}, \mathrm{H}_{2}^{+}, \mathrm{H}^{-}$ & $\mathrm{No}^{d}$ & $\mathrm{H}_{2}$ \\
Classical Ions & Yes & Yes & Yes & Yes \\
Electron Degeneracy & relativistic & nonrelativistic & nonrelativistic & relativistic \\
Excited States & No & Yes & Yes & No \\
Electron Exchange & No & No & Yes & Yes \\
Quantum Diffraction & No & No & Yes & No \\
\hline
\end{tabular}

${ }^{a}$ Debye-Hückel approximation

${ }^{b}$ ad hoc

${ }^{c}$ ad hoc, but more general than in EFF

${ }^{d}$ Molecules will be included in future OPAL tables

(http://visier.u-strasbg.fr/OP.html). MHD tables adequate for solar and stellar modeling can be directly obtained from W. Däppen (e-mail: dappenQusc . edu).

\section{b) OPAL EOS}

The OPAL (Rogers et al. 1996) EOS is based on the physical picture. See Table 1 below for a summary of the physics included. Although OPAL EOS can include molecules in principle, the OPAL tables commonly used do not include molecules, and extend to $T>6000 \mathrm{~K}$. These tables must then be supplemented to calculate, for example conditions near the solar photosphere. Like MHD, OPAL does not include relativistic effects. These EOS tables are valid for stars of mass $\geq 0.8 \mathrm{M}_{\odot}$ on or above main sequence. The tables are available for fixed $\mathrm{Z}$ between 0.0 and 0.04 , and $\mathrm{X}=0$ to 0.8 . The element mixture includes $\mathrm{H}, \mathrm{He}, \mathrm{C}, \mathrm{N}, \mathrm{O}$, and $\mathrm{Ne}$, derived from the Grevesse \& Noels (1991) solar abundances.

See the web site http://www-phys.Ilnl.gov/V.Div/OPAL/ for tables, interpolation routines and additional information.

\subsection{RADIATIVE OPACITIES: MID- TO HIGH TEMPERATURES}

\subsubsection{Analytical Fits to Tables}

Analytical fits to opacity tables were popular in the 1960s through $1980 \mathrm{~s}$, but unfortunately no new analytical fits have been published since the advent of the OPAL and OP opacity tables in the early 1990s. Analytical fits can be very useful, for reasons described in the introduction, such as 
smooth derivatives, avoiding interpolation and extrapolation, applicability to a larger range of stellar conditions, more flexibility in modifications, etc. and it would be worthwhile to derive new fits to the modern tables. For some applications, old analytical fits have been re-calibrated using selective multipliers to agree approximately with new table values (see, e.g., Guzik $\&$ Cox 1995).

a) Iben and Christy analytical fits

Iben (1975) developed an analytical fit to the Cox \& Stewart (1970a,b) opacity tables for his general stellar evolution code. For temperatures less than 1 million K, Iben applied the Christy (1966) analytical fit.

b) Stellingwerf analytical fit

Stellingwerf (1975a) describes his analytical fit to Los Alamos opacity tables King Ia (X, Z = 0.7, 0.001) and King IIa (X, $Z=0.8,0.001)$ generated by Cox et al. (1973). An erratum to this opacity fit appears as a footnote in Stellingwerf (1975b).

\subsubsection{Tables}

a) LAOL

The opacity tables of the Los Alamos Opacity Library (Huebner et al. 1977) were the standard for many years, but proved to be inadequate, especially for Cepheids and other variables, and the solar interior (see Rogers \& Iglesias 1994). The OPAL and OP projects found opacity increases over the LAOL values by as much as a factor of three for stellar envelope conditions near a few hundred thousand $\mathrm{K}$ with Pop. I abundances. The main contributors to higher opacities were bound-bound absorptions in Fe. The OPAL opacities are also about $20 \%$ higher in the 2 to 5 million $\mathrm{K}$ region below the solar convection zone. This opacity increase deepened the solar convection zone, improving agreement with its helioseismically-determined depth (see, e.g., Guzik \& Cox 1991).

b) OPAL

The OPAL tables (Iglesias \& Rogers 1996; Rogers \& Iglesias 1992; Iglesias et al. 1992) are based on the Grevesse \& Noels (1991) solar element mixture, collapsed to 14 elements. The updated tables of Iglesias \& Rogers (1996) are based on the Grevesse \& Noels (1993) mixture, incorporating 21 elements, and in addition are calculated using an improved EOS treatment. The tables span the range $3.75 \leq \log T \leq 8.7$, and $-8 \leq \log R \leq+1$, where $R=T_{6}^{3} / \rho$ and $T_{6}=10^{-6} T$. Tables are also available for enhanced $\alpha$-element mixtures, and enhanced $\mathrm{CO}$ mixtures. Opacity tables may also be requested for any desired element mixture. 
These opacities and interpolation routines are available on the web site http://www-phys.1lnl.gov/V Div/OPAL/

c) LEDCOP

Los Alamos has generated new opacities, using the so-called LEDCOP (Los Alamos Light Element Detailed Configuration Opacity) code (Magee et al. 1995). The new Los Alamos Astrophysical Opacity database contains elements from Hydrogen to Zinc (atomic number $Z=1-30$ ). One can use the so-called TOPS code (Abdallah \& Clark) (1985) to manipulate the individual element data to generate Rosseland or Planck mean opacities for any desired mixture. The user can choose the $\rho$ grid, but the $T$ grid is fixed. The ranges of validity in $\mathrm{T}$ and $\rho$ are $0.5 \mathrm{eV}<T<100 \mathrm{keV}$, and $10^{-10} \leq \rho \leq 10^{9} \mathrm{~g} \mathrm{~cm}^{-3}$. The EOS for these opacities is based on the Saha equation, including degeneracy and other refinements. The Saha equation is solved iteratively to obtain a consistent set of ion abundances, bound state occupancies, and free electrons.

These codes and tables are available on the web site http://t4.Ianl.gov. Tables for the GN93 solar mixture and other mixtures of interest will be available on the web site shortly. These new Los Alamos opacities have not been extensively tested for astrophysical applications, but direct comparisons with OPAL opacities shows that the agreement is much closer than between OPAL and the older LAOL opacities.

d) $\mathrm{OP}$

The Opacity Project (see the comprehensive books by Seaton 1995 and Berrington 1997) provides monochromatic opacities for 17 elements up through $\mathrm{Ni}$, on grids of $T$ and $N_{e}$ (electron density). Tables of Rosseland and Planck mean opacities for 213 fixed $X, Z$ compositions using the element mixtures listed in Seaton et al. (1994) are also available. Codes are provided to interpolate to any value of density and temperature, and also to interpolate in X and $\mathrm{Z}$. These opacities are generated using the MHD EOS. The web site (see below) advertises that data will be available shortly for calculating radiative forces, which would be useful for calculating radiative levitation of elements to compare with surface abundances of peculiar A and F stars. Monochromatic opacities for each element are done, so tables can be requested for any desired mixture. The drawback of these tables is the limited range of validity compared to OPAL: $-7<\log R<-1$, and $3.5 \leq \log T \leq 7.0$. However, for densities less than $0.01 \mathrm{~g} \mathrm{~cm}^{-3}$ care should be taken, since dense plasma effects not included in OP may become important. Codes are available to fit, smooth, and interpolate between both OP and OPAL opacity tables (Seaton 1993, 1996).

Codes and tables are available on the web site of the Opacity Project http://visier.u-strasbg.fr/OP.html. For more information, contact 
Anil Pradhan (e-mail: pradhanQastronomy.ohio-state.edu) He reports that his group and other members of the Iron Project are recalculating radiative data for $\mathrm{Fe}$, which should greatly improve accuracy over OPAL and $O P$ results.

e) Comparison of OPAL, LEDCOP, and OP opacities

Figure 1 compares the OPAL (LLNL), LEDCOP (latest LANL), and OP opacities for stellar interior conditions. These modern tables agree quite well with each other, but small differences remain. For example, for conditions at the base of the solar convection zone, which determine the convection zone depth, the LEDCOP opacities are 1 to $5 \%$ less than OPAL, and about $30 \%$ larger than OP.

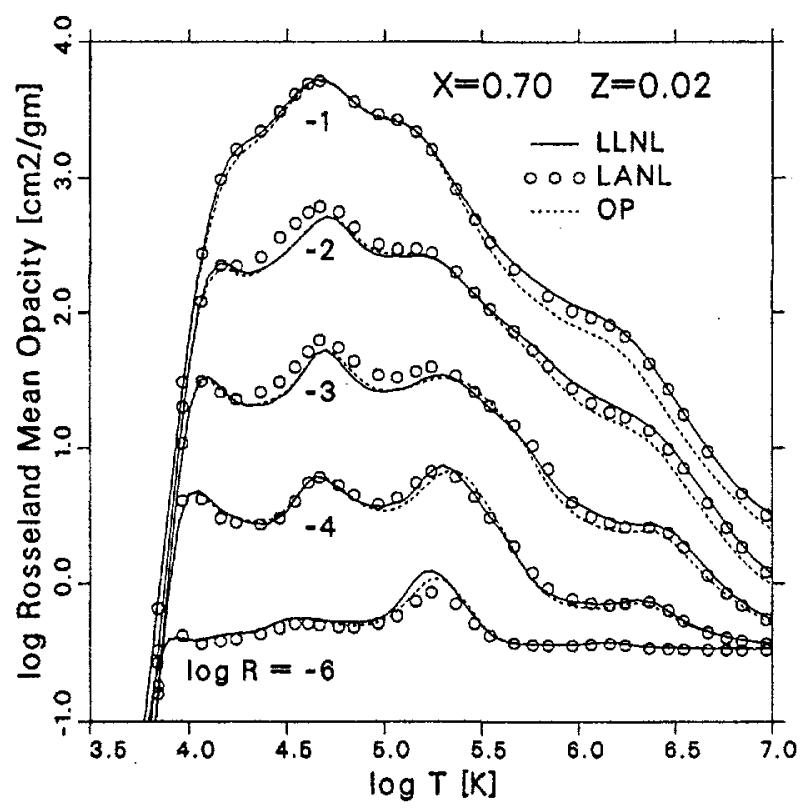

Figure 1. OPAL (LLNL), LEDCOP (latest LANL), and OP Rosseland mean opacities versus temperature for $\mathrm{X}=0.7$ and $\mathrm{Z}=0.02$, for several $\log R$ values, where $R=\rho / T_{6}^{3}$. Figure provided by J. J. Keady (LANL).

\subsection{RADIATIVE OPACITIES: LOW TEMPERATURES}

Note that neither the OPAL nor OP opacities extend to low temperatures or include molecules. Also, it may be more correct to use Planck mean opacities for optically thin layers. For this reason, modelers of the Sun and lowmass stars must supplement the OPAL or OP tables with low-temperature 
opacities. Some of the more recent and popular low-temperature tables and fits are listed below.

\subsubsection{Kurucz}

Kurucz (1992) provides Rosseland mean opacities including 58 million lines and diatomic molecules for $1>\log [Z]>-3$ relative to solar, based on the Anders \& Grevesse (1989) mixture. These tables include 56 temperatures ranging from 2000 to $20,000 \mathrm{~K}, 21 \log P$ values from -2 to 8 , and at least two $Y$ compositions 0.25 and 0.31 . Tables are also available for several different microturbulent velocities $0,1,2,4$ and $8 \mathrm{~km} / \mathrm{sec}$. More information and tables are available at the web site http://cfaku5.harvard.edu/OPACITIES.

\subsubsection{Alexander $\&$ Ferguson}

Alexander \& Ferguson [1994 (Grevesse \& Noels 1991 solar mixture); 1995, (Grevesse \& Noels 1993 solar mixture)] provide Rosseland and Planck mean opacity tables valid for $T=700-12,500 \mathrm{~K}$ including atomic and molecular line absorption, grain absorption and scattering by silicates, iron, carbon, and SiC. Alexander \& Ferguson (1994) find that including molecules becomes necessary for $T<5000 K$, tri-atomic molecules for $T<3200 K$, and grains for $T<1700 \mathrm{~K}$. These opacities are available from Alexander (e-mail: dra@twsuvm.uc.twsu.edu)

\subsubsection{Neuforge}

Neuforge (1993) generated Rosseland mean opacity tables using the Anders \& Grevesse (1989) solar mixture. These are valid for $T=3000-15,000 \mathrm{~K}$ and $-0.383<Q<4.605$, where $Q=\ln \left(\rho^{1 / 3} / T_{7}\right)$, and $T_{7}=T / 10^{7}$. These opacities include a subset of $\mathrm{H}, \mathrm{CI}, \mathrm{MgI}, \mathrm{AlI}, \mathrm{SiI}$, bound-bound and freefree transitions, and line absorption for some neutral atoms and molecules. The tables are produced for $\mathrm{X}=0,0.2,0.5,0.7,0.9$ and $\mathrm{Z}=0.0001,0.001$, $0.005,0.02,0.04$. Even though Kurucz (1992) includes millions of lines, these opacities are within $13 \%$ of Kurucz values. The tables are available from Neuforge (e-mail: neuforge@lanl.gov, neuforge@astro.ulg.ac.be).

\subsubsection{Sharp}

Sharp (1992) produced Planck and Rosseland mean molecular opacity tables for a solar (Cameron 1973) mixture, and for enhanced CNO abundances. Sharp generated the enhanced CNO opacities for application to accretion disks of cataclysmic binaries due to nova eruption of the white dwarf, and finds a significant opacity increase between 3000 and $6000 \mathrm{~K}$ over the normal CNO tables. The range of applicability is $k_{B} T=0.2-1 \mathrm{eV}$ 
and $10^{-10}<\rho<10^{-3} \mathrm{~g} \mathrm{~cm}^{-3}$. Sharp assumes a turbulent broadening of 1 $\mathrm{km} / \mathrm{s}$.

\subsubsection{Bell}

Bell (1993) calculated Rosseland mean opacities, including atomic lines and 12 molecules.

\subsubsection{Another option}

A final option, if an analytical opacity is desired, is to use the Stellingwerf $(1975 a, b)$ or Iben (1975) analytical opacities, and modify some of the terms or add multipliers to calibrate the fit to the low-temperature tables (see, e.g., Guzik \& Cox 1991, 1995).

\subsection{CONDUCTIVE OPACITIES}

Electron heat conduction is important when free electron densities are high, and electrons begin to become degenerate. This heat conduction can be incorporated into the radiative transfer solution as a "conductive opacity" and added in reciprocal to the radiative opacity. This opacity is normally very high, so that it modifies the radiative opacity by only a few percent in the core of the sun and main-sequence stars. However, conductive opacities must not be neglected, even for solar interior calculations. Electron conduction becomes increasingly important as the star evolves, and dominates energy transport for stars with significant degeneracy, e.g. in white dwarfs and the deep interior of some red supergiants.

\subsubsection{Canuto}

The Canuto et al. (1970) conductive opacities are used by Iben (1975) in his general stellar evolution code for densities greater than $2 \times 10^{6} \mathrm{~g} \mathrm{~cm}^{-3}$.

\subsubsection{Hubbard \& Lampe}

The Hubbard \& Lampe (1969) conductive opacities are used by Iben (1975) in his general stellar evolution code for densities less than $10^{6} \mathrm{~g} \mathrm{~cm}^{-3}$. For $6<\log \rho<6.3$, Iben (1975) adopts a weighted average of these conductive opacities and the Canuto conductive opacities.

\subsubsection{Itoh et al.}

The Itoh \& Kohyama (1993) and Itoh et al. $(1983,1984,1993,1994)$ conductive opacities are more recent, and have been popular for white dwarf evolution and pulsation calculations. 


\subsection{EQUATION OF STATE AND OPACITY FOR DEGENERATE MATTER}

Finally, the above list of radiative opacities and equations of state is inadequate for objects with significantly degenerate matter, such as white dwarfs, brown dwarfs, and giant planets. For white dwarfs, OPAL opacities are available for for appropriate $\mathrm{CO}$ mixtures for interiors, and $\mathrm{H}$ and $\mathrm{He}$ for envelopes (Iglesias \& Rogers 1993). These tables need to be supplemented by other opacities, e.g. the LAOL opacities, at the highest temperatures and densities.

For white dwarfs, the Fontaine, Graboske \&.Van Horn (1977) EOS for $\mathrm{H}, \mathrm{He}$, and $\mathrm{CO}$ mixtures is commonly used at moderate densities, and is supplemented by the Lamb (1974) tables for high density and CO mixtures (see also Lamb 1975). The Saumon, Chabrier \& Van Horn (1995) EOS tables are popular for low-mass stars and giant planets.

\subsection{OPACITY AND EOS COMPARISONS AND EVALUATIONS}

Below we list some papers that compare results for solar and stellar models using different EOS or opacity treatments, or evaluate opacity or EOS treatments in light of helioseismic or other observations. This list is by no means exhaustive.

\subsection{1. $E O S$}

- Comparison of solar models using the Ezer versus MHD EOS (Yildiz \& Kiziloglu 1997)

- Effect of relativistic corrections on adiabatic exponent $\Gamma_{1}$ and solar core structure (Elliot \& Kosovichev 1998)

- EFF EOS results shown to be inferior to modern EOSs for solar soundspeed inversions (Basu \& Christensen-Dalsgaard 1997)

- EFF versus CEFF versus MHD EOS for solar models (ChristensenDalsgaard \& Däppen 1992; Christensen-Dalsgaard 1991)

- Validation of MHD EOS for low-mass stars 0.4-1M $\odot$ (Charbonnel et al. 1999)

- Comparison of solar models using MHD versus older equations of state (Guzik \& Cox 1991)

- MHD versus OPAL versus SIREFF for solar models (Guzik \& Swenson 1997)

- MHD versus OPAL for solar models (Guenther et al. 1996)

- Inclusion of excited states in hydrogen internal partition function, and signature in solar oscillations inversions (Nayfonov \& Däppen 1998)

- Effects of electron exchange correction on solar oscillations (Guzik \& Swenson 1997) 
- Effect of including $\mathrm{Z}$ variation due to diffusion in EOS on solar structure (Guzik \& Swenson 1997)

- MHD EOS preferred over OPAL EOS for outer $2 \%$ of solar radius (Basu et al. 1999)

\subsubsection{Opacities}

- Comparison of solar models using Cox \& Stewart versus OPAL opacities (Yildiz \& Kiziloglu 1997)

- Advantages of OPAL over LAOL opacities (Rogers \& Iglesias 1994)

- Comparison of OPAL to OP and Alexander \& Ferguson (1994) opacities (Iglesias \& Rogers 1996)

- Discrepancies between OPAL and OP at high densities and temperatures (Iglesias \& Rogers 1995)

- Comparison of several low-temperature opacity sets (Neuforge 1993)

- Effect of low-temperature opacities on solar models and p-mode frequencies (Gong \& Däppen 1998)

- Comparison of 1991 versus 1993 OPAL opacities for solar oscillations (Guzik \& Swenson 1997)

- OPAL versus OP opacities for solar models (Gong et al. 1998; Gong \& Li 1998)

- Effect on solar models of localized interior opacity changes (Brun et al. 1998; Gabriel 1997)

- Comparison of evolutionary tracks for OPAL and LAOL opacities for stars of 1 to $7 \mathrm{M}_{\odot}$ (Cassisi et al. 1994)

- Fitting, smoothing, and interpolating between tables of opacity data (Seaton 1993, 1996)

- Testing of opacity interpolation schemes using a similar analytical formula (Christensen-Dalsgaard \& Däppen 1992)

- Note on a new interpolation scheme for opacity tables (Houdek \& Rogl 1993)

\section{Conclusion}

As far as the equation of state is concerned there has not only been substantial theoretical progress in the last decades, but the spectacular observational accuracy of helioseismology has constrained the plasma of the solar interior so strongly that rather fine deviations from the simple gas models are now observationally well established. For instance, the DebyeHückel Coulomb pressure correction is now routinely seen in helioseismic data. However, even finer effects are becoming accessible. They are the targets of new ground and space missions, dedicated to helioseismology, which have begun to improve the already good observational situation. 
As far as the opacity is concerned, the new data of the last few years has had a very favorable impact on the astrophysical explanation of a broad range of stellar properties. This success provides a strong motivation to extend the calculations to cover a broader range of applications. For example, the temperature and density range of white dwarfs or other dense stellar objects are partly beyond the range of the current tables, the elemental composition is not adequate to model s-process stars that have significant amounts of elements heavier than $\mathrm{Fe}$, and there are many applications requiring frequency dependent opacity data such as radiative levitation (Seaton 1997; Richer et al. 1997).

In the specific case of the Sun, current opacity tables only allow for changes in the total $Z$. To facilitate the process of adding diffusion to the best standard solar models (SSM) (see Christensen-Dalsgaard 1998) it will be necessary to provide opacity tables that allow for variation of individual element abundances. Due to the stringent requirements set by helioseismology, and with likely progress in the helioseismic abundance determinations, even small sources of opacity not included in the current calculations will need to be considered. Ultimately, this will make the Sun a formidable opacity experiment.

\section{Acknowledgments}

We thank Forrest Rogers and Alan Nayfonov for providing information for the equation of state and opacity sections. We thank Fritz Swenson, Paul Bradley, Norm Magee, Anil Pradhan, John Keady, and J. ChristensenDalsgaard for providing information for the recommendations section. W.D. is supported by the grant AST-9618549 of the National Science Foundation, by the SOHO Guest Investigator Grant NAG5-7352 of NASA, and by the Danish National Research Foundation through its establishment of the Theoretical Astrophysics Center. J.A.G. acknowledges financial support from the Dept. of Energy and NASA Astrophysics Theory Program grant S-30934-F. We both thank Cafer Ibanoglu and Can Akan, as well as their superb team, for organizing an outstanding interdisciplinary meeting. 


\section{References}

Abdallah, J., Jr. \& Clark, R.E.H. 1985, TOPS: A Multigroup Opacity Code, Los Alamos Report LA-10454.

Alastuey, A. \& Perez, A. 1992, Europhys. Lett., 20, 19-24.

Alastuey, A. \& Perez, A. 1996, Phys. Rev. E, 53, 5714.

Alastuey, A., Cornu, F. \& Perez, A. 1994, Phys. Rev. E, 49, 1077.

Alastuey, A., Cornu, F. \& Perez, A. 1995, Phys. Rev. E, 51, 1725.

Alexander, D.R. \& Ferguson, J.W. 1994, Astrophys. J., 437, 879

Antia H.M. 1998, A\&A, 330, 336.

Anders, E. \& Grevesse, N., 1989, Geochim. Cosmochim. Acta. 53, 197.

Bahcall \& Glasner 1994, Astrophys. J., 437, 484.

Basu, S. \& Christensen-Dalsgaard, J. 1997, Astron. Astrophys., 322, L5-L8.

Basu, S., Däppen, W. \& Nayfonov, A. 1999, Astrophys. J., in press.

Baturin, V.A., Däppen, W., Wang, X. \& Yang, F. 1996, in Proc. 32nd Liège International Astrophysical Colloquium "Stellar Evolution: What Should be Done", ed. M. Gabriel \& A. Noels (Liège: Institut d'Astrophysique), 33.

Bautista, M.A. \& Pradhan, A.K.: 1997, Astron. Astrophys., 126, 365.

Bell, R.A. 1993, B.A.A.S., 183, 111.07.

Berrington, K.A. 1995, The Opacity Project, vol. II, (Bristol: Institute of Physics Publishing).

Boercker, D.B.: 1987, Astrophys. J., 316, L95.

Brüggen, M. \& Gough, D.O. 1999, Phys. Rev. E, submitted.

Brun, A.S., Turck-Chieze, S. \& Morel, P. 1998, Astrophys. J., 506, 913.

Cameron, A.G.W. 1973, Space Science Reviews, 15, 121.

Canuto, V. 1970, Astrophys. J., 159, 641.

Cassisi, S., Castellani, V., Salaris, M. \& Straniero, O. 1994, Astron. Astrophys., 282, 760.

Cauble, R., Perry, T.S., Bach, D.R., Budil, K.S., Hammel, B.A. et al. 1998, Phys. Rev. Letters, 80, 1248.

Charbonnel, C., Däppen, W., Schaerer, D., Bernasconi, P.A., Maeder, A., Meynet, G., Mowlavi, N. 1999, Astron. Astrophys. Suppl. Ser., 135, 405.

Christensen-Dalsgaard, J, 1991, in Lecture Notes in Physics, Vol, 388: Challenges to Theories of the Structure of Moderate-mass Stars, ed. D.O. Gough \& J. Toomre (Heidelberg: Springer), 11.

Christensen-Dalsgaard, J. 1998, Space Science Reviews, 85, 19-36.

Christensen-Dalsgaard, J. \& Däppen, W. 1992, Astron. Astrophys. Review, 4, 267.

Christensen-Dalsgaard, J. \& Dziembowski, W.A., 1999. (Basic aspects of stellar structure and pulsation]. This volume (Chapter I).

Christensen-Dalsgaard, J., Däppen, W. \& Lebreton, L. 1988, Nature, 336, 634.

Christensen-Dalsgaard, J., Däppen, W., Dziembowski, W.A. \& Guzik, J.A., 1999. [An introduction to helioseismology]. This volume (Chapter II).

Christy, R.F. 1966, Astrophys. J., 144, 108.

Cox, A.N. 1965 in Stars and Stellar Systems, Vol. 8, eds. I.H. Aller \& D.R. McLaughlin (Chicago: University of Chicago Press), 195-267.

Cox, A.N.: 1991, Astrophys. J., 381, L7138.

Cox, J.P. \& Giuli, R.T. 1968, Principles of Stellar Structure (NewYork: Gordon \& Breach)

Cox, A.N. \& Stewart, J.N. 1962, Astrophys. J., 67, 113.

Cox, A.N. \& Stewart, J.N. 1965, Astrophys. J. Suppl., 11, 22.

Cox, A.N. \& Stewart, J.N. 1970a, Astrophys. J. Suppl., 19, 243.

Cox, A.N. \& Stewart, J.N. 1970b, Astrophys. J. Suppl., 19, 261.

Cox, A.N. \& Tabor, J.E. 1976, Astrophys. J. Suppl., 31, 271.

Cox, A.N., King, D.S. \& Tabor J.E. 1973, Astrophys. J., 184, 201.

Däppen, W. 1980, Astron. Astrophys., 91, 212.

Däppen, W. 1998, Space Science Reviews, 85, 49-60.

Däppen, W., Anderson, L.S. \& Mihalas, D. 1987, Astrophys. J., 319, 195. 
Däppen, W., Keady, J. \& Rogers, F. 1991, in The Solar Interior and Atmosphere, edited by A.N. Cox, W. Livingston \& R. Mathews (University of Arizona Press, Tucson, Ariz., 1991), 112-139.

Däppen, W., Mihalas, D., Hummer, D.G. \& Mihalas, B.W. 1988, Astrophys. J., 332, 261.

Debye, P. \& Hückel, E. 1923, Z. Phys., 24, 305.

Diesendorf, M.O. \& Ninham, B.W. 1969, Astrophys. J., 156, 1069.

Dimitrievic, M.S. \& Konjevic, N. 1980, J. Quant. Spectrosc. Rad. Transf. 24, 451.

Ebeling, W., Kraeft, W.D. \& Kremp, D. 1976, Theory of Bound States and Ionization Equilibrium in Plasmas and Solids (DDR-Berlin: Akademieverlag)

Ebeling, W., Förster, A., Fortov, V.E., Gryaznov, V.K. \& Polishchuk, A.Ya. 1991, Thermodynamic Properties of Hot Dense Plasmas (Stuttgart: Teubner)

Eggleton, P.P., Faulkner, J. \& Flannery, B.P. 1973, Astron. Astrophys, , 23, 325.

Elliot J.R. \& Kosovichev, A.G 1998, Astrophys. J., 500, L199.

Fowler, R.H. 1936, Statistical Mechanics (Cambridge: Cambridge University Press)

Foley, H.M. 1946, Phys. Rev., 69, 616-628.

Fontaine, G., Graboske, H.C., Jr. \& Van Horn, H.M. 1977, Astrophys. J. Suppl., 35, 293.

Fricke, K., Stobie, R.S. \& Strittmatter, P.A. 1971, Mon. Not. R. astr. Soc., 154, 23.

Gabriel, M. 1997, Astron. Astrophys., 327, 771.

Gong, Z. \& Däppen, W. 1998, in SOHO6/GONG98 Workshop, Structure and Dynmaics of the Interior of the Sun and Sun-like Stars, Boston (ESA SP-418), 465.

Gong, Z. \& Li, Y. 1998, in IAU Symp. 181, Sounding Solar and Stellar Interiors, eds. J. Provost \& F.X. Schmider, 91.

Gong, G., Däppen, W. \& Li, Y. 1998, in Cool Stars, Stellar Systems and the Sun, ASP Conf. Ser. Vol. 154, eds. R.A. Donahue \& J.A. Bookbinder, 266.

Graboske, H.C., Harwood, D.J. \& Rogers, F.J. 1969, Phys. Rev., 186, 210.

Grevesse, N. \& Noels, A. 1991, Astron. Astrophys., 242, 488.

Grevesse N., Noels A. 1993, in Origin and Evolution of the Elements, ed. Prantzos N., Vangioni-Flam E. \& Cassé M., (Cambridge: Cambridge University Press), 15.

Griem, H.R.: 1960, Astrophys. J., 132, 883.

Griem, H.R., Baranger, M., Kolb, A.C. \& Oertel, G. 1962, Phys. Rev., 125, 126-141.

Guenther, D.B., Kim, Y.-C. \& Demarque, P. 1996, Astrophys. J., 463, 382.

Guzik, J.A. \& Swenson, F.J. 1997, Astrophys. J., 491, 967.

Guzik, J.A. \& Cox, A.N. 1991, Astrophys. J., 381, 333.

Guzik, J.A. \& Cox, A.N. 1995, Astrophys. J., 448, 905.

Harris, G.M. 1959, J. Chem. Phys., 31, 1211.

Harris, G.M. 1962, Phys. Rev., 125, 1131.

Harris, G.M., Roberts, J.E. \& Trulio, J.G. 1960, Phys. Rev., 119, 1832.

Holtsmark, J. 1919, Ann. Phys., 58, 577.

Houdek, G. \& Rogl, J., 1993 Communications in Asteroseismology, ed. W. W. Weiss, No. 60.

Hubbard, W.B. \& Lampe, M. 1969, Astrophys. J. Stuppl., 18, 297.

Huebner, W.F. 1986, in Physics of the Sun, ed. P.A. Sturrock, T.E. Holzer, D.M.Mihalas \& R.K. Ulrich (Dordrecht: Reidel), Vol. 1, 33.

Huebner, W.F., Merts, A.L., Magee, N.H. \& Argo, M.F. 1977, Los Alamos Scientific Report LA-6760-M.

Hui, A.K., Armstrong, B.H. \& Wray, A.A. 1978, J. Quant. Spectrosc. Rad. Transf. 19, 509.

Hummer, D.G. \& Mihalas, D. 1988, Astrophys. J., 331, 794.

Iben, I., Jr. 1975, Astrophys. J., 196, 525.

Iglesias, C.A. \& Rogers, F.J., 1991, Astrophys. J., 371, 408.

Iglesias, C.A. \& Rogers, F.J., 1993, Astrophys. J., 412, 752.

Iglesias, C.A. \& Rogers, F.J. 1995, Astrophys. J., 443, 460.

Iglesias, C.A. \& Rogers, F.J. 1996, Astrophys. J., 464, 943.

Iglesias, C.A., Rogers, F.J. \& Wilson, B.G. 1987 Astrophys. J. Lett., 322, L45. 
Iglesias, C.A., Rogers, F.J., \& Wilson, B.G. 1992, Astrophys. J., 397, 717.

Iglesias, C.A., DeWitt, H.E., Lebowitz, J.L., MacGowan, D. \& Hubbard, W.B.: 1985, Phys. Rev. A., 31, 1698.

Irwin, A., Swenson, F.J., VandenBerg, D., \& Rogers, F.J. 1999, in preparation

Itoh, N. \& Kohyama, Y. 1993, Astrophys. J., 404, 268.

Itoh, N., Hayashi, H., \& Kohyama, Y. 1993, Astrophys. J., 418, 405.

Itoh, N., Hayashi, H., \& Kohyama, Y. 1994, Astrophys. J., 438, 418.

Itoh, N., Kohyama, Y., Matsumoto, N., \& Seki, M., 1984, Astrophys. J., 285, 758.

Itoh, N., Mitake, S., Iyetomi, H. \& Ichimaru, S. 1983, Astrophys. J., 273, 774.

Kraeft W.D., Kremp, D., Ebeling, W. \& Röpke G. 1986, Quantum Statistics of Charged Particle Systems (New York: Plenum)

Kurucz, R.L. 1992, Revista Mexicana de Astronomía y Astrofísica, 23, 181.

Lamb, D.Q. 1974, Ph.D. thesis, (Rochester: Univ. Rochester)

Lamb, D.Q. \& Van Horn, H.M. 1975, Astrophys. J., 200, 306.

Lee, R.W.: 1988, J. Quant. Spectrosc. Rad. Transf. 40, 561.

Lindholm, E. 1946, Ark. Mat. Astron. Phys., 32a, 17.

Magee, N.H. \& Clark, R.E.H. 1998, in Proc. of the International Conference on Atomic and Molecular Data and their Applications, Gaithersburg, Maryland, in press.

Magee, N.H., Merts, A.L. \& Huebner, W.F. 1984, Astrophys. J., 283, 264.

Magee, N.H. Jr., Abdallah, J., Jr., Clark, R.E.H., Cohen, J.S., Collins, L.A., Csanak, G., Fontes, C.J., Gauger, A., Keady, J.J., Kincrease, D.P. \& Merts, A.L. 1995, in Astrophysical Applications of Powerful New Databases, eds. S.J. Adelman \& W.L. Wiese (ASP Conf. Ser. 78), 51.

Mihalas, D., Däppen, W. \& Hummer, D.G. 1988, Astrophys. J., 331, 815.

Nayfonov, A. \& Däppen, W. 1998, Astrophys. J., 499, 489.

Nayfonov, A., Däppen, W., Hummer, D.G. \& Mihalas, D.M. 1999, Astrophys. J., in press. Neuforge, C. 1993, Astron. Astrophys., 274, 818.

Perez, A. \& Däppen, W. 1999, Astrophys. J., in preparation.

Petersen, J.O.: 1974, Astron. Astrophys., 34, 309.

Reichl, L.E. 1980, A Modern Course in Statistical Physics (Austin: University of Texas Press).

Richer, J., Michaud, M.G., Rogers, F., Iglesias, C., Turcotte, S. \& LeBlanc, F. 1997, Astrophys. J., 492, 833 .

Rogers, F.J. 1977, Phys. Lett., 61A, 358.

Rogers, F.J. 1981, Phys. Rev., A24, 1531.

Rogers, F.J. 1986, Astrophys. J., 310, 723-728.

Rogers, F.J. 1991, in High Pressure Equations Of State: Theory and Applications (ed. by S. Eliezer \& R.A. Ricci, North Holland, New York)

Rogers, F.J. 1994, in The Equation of state in Astrophysics (IAU Colloquium 147, ed. G. Chabrier \& E. Schatzman, Cambridge University Press, 1994), 16-42.

Rogers, F.J. 1998, Space Science Reviews, 85, 61-70.

Rogers, F.J. \& Iglesias, C.A., 1992 Ap.J. Supp. 401, 361.

Rogers, F.J. \& Iglesias, C.A., 1994, Science 263, 50.

Rogers, F.J., Swenson, F.J. \& Iglesias, C.A. 1996, Astrophys. J., 456, 902.

Rogers, F.J., Wilson, B.G. \& Iglesias, C.A. 1988, Phys. Rev. A38, 5007.

Ross, J.E. \& Aller, L.H. 1976, Science, 191, 1223.

Saumon, D., Chabrier, G., \& Van Horn, H.M. 1995, Astrophys. J. Suppl., 99, 713.

Seaton, M.J. 1987, J. Phys. B: Atom. Molec. Phys., 20, 6363-6378.

Seaton, M.J. 1988, J. Phys. B.: Atom. Molec. Phys., 21, 3033.

Seaton, M.J. 1992, Revista Mexicana de Astronomía y Astrofísica, 23, 180.

Seaton, M.J. 1993, Mon. Not. R. astr. Soc., 265, L25.

Seaton, M.J. 1995, The Opacity Project Vol. I, (Bristol: Institute of Physics Publishing)

Seaton, M.J. 1996, Mon. Not. R. astr. Soc., 279, 95.

Seaton, M.J. 1997, Mon. Not. R. astr. Soc., 289, 700.

Seaton, M.J., Yan, Y., Mihalas, D. \& Pradhan, A.K. 1994, Mon. Not. R. astr. Soc., 266, 
805.

Sharp, C.M. 1992, Astrophys. J. Suppl., 94, 1.

Stellingwerf, R.F. 1975a, Astrophys. J., 195, 441.

Stellingwerf, R.F. 1975b, Astrophys. J., 199, 705.

Stellingwerf, W.F. 1978, Astrophys. J., 83, 1184.

Simon, N.R. 1982, Astrophys. J. Lett., 260, L87.

Tripathy, S.C., Basu, S., \& Christensen-Dalsgaard, J. 1997, in Proc. of IAU Symp. 181, eds. Schmider, F.X. \& Provost, J., 129.

Vardya, M.S. 1964, Am. J. Phys., 32, 520 .

Wiese, W.L., Kelleher, D.E. \& Paquette, D.R.: 1972, Phys. Rev. A6, 1132.

Watson, W.D. 1969, Astrophys. J., 157, 375.

Yildiz, M. \& Kiziloglu, N. 1997, Astron. Astrophys., 327, 187. 